\title{
Stimulation in the Rat Anterior Insula and Anterior Cingulate During an Effortful Weightlifting Task
}

\author{
Carlos Silva, Blake S. Porter and Kristin L. Hillman* \\ Department of Psychology, Brain Health Research Centre, University of Otago, Dunedin, New Zealand
}

\section{OPEN ACCESS}

Edited by:

Nicolas Silvestrini,

Université de Genève, Switzerland

Reviewed by:

Veit Stuphorn,

Johns Hopkins University,

United States

Michael X. Cohen,

University of Amsterdam, Netherlands

*Correspondence:

Kristin L. Hillman

kristin.hillman@otago.ac.nz

Specialty section

This article was submitted to

Decision Neuroscience,

a section of the journal

Frontiers in Neuroscience

Received: 18 December 2020

Accepted: 11 February 2021

Published: 26 February 2021

Citation:

Silva C, Porter BS and Hillman KL (2021) Stimulation in the Rat Anterior Insula and Anterior Cingulate During

an Effortful Weightlifting Task.

Front. Neurosci. 15:643384.

doi: 10.3389/fnins.2021.643384
When performing tasks, animals must continually assess how much effort is being expended, and gage this against ever-changing physiological states. As effort costs mount, persisting in the task may be unwise. The anterior cingulate cortex (ACC) and the anterior insular cortex are implicated in this process of cost-benefit decision-making, yet their precise contributions toward driving effortful persistence are not well understood. Here we investigated whether electrical stimulation of the ACC or insular cortex would alter effortful persistence in a novel weightlifting task (WLT). In the WLT an animal is challenged to pull a rope $30 \mathrm{~cm}$ to trigger food reward dispensing. To make the action increasingly effortful, $45 \mathrm{~g}$ of weight is progressively added to the rope after every 10 successful pulls. The animal can quit the task at any point - with the rope weight at the time of quitting taken as the "break weight." Ten male Sprague-Dawley rats were implanted with stimulating electrodes in either the ACC [cingulate cortex area 1 (Cg1) in rodent] or anterior insula and then assessed in the WLT during stimulation. Lowfrequency $(10 \mathrm{~Hz})$, high-frequency $(130 \mathrm{~Hz})$, and sham stimulations were performed. We predicted that low-frequency stimulation (LFS) of $\mathrm{Cg} 1$ in particular would increase persistence in the WLT. Contrary to our predictions, LFS of Cg1 resulted in shorter session duration, lower break weights, and fewer attempts on the break weight. Highfrequency stimulation of Cg1 led to an increase in time spent off-task. LFS of the anterior insula was associated with a marginal increase in attempts on the break weight. Taken together our data suggest that stimulation of the rodent $\mathrm{Cg} 1$ during an effortful task alters certain aspects of effortful behavior, while insula stimulation has little effect.

Keywords: effort, insula, cingulate cortex, persistence, rat, weightlifting

\section{INTRODUCTION}

The ability to appropriately persevere or abandon effortful tasks is essential for optimal function (Hull, 1943). Persisting through effort is often needed to achieve highly valued rewards, but organisms must also know when to quit behaviors or tasks that are no longer optimal based on external and/or internal signals (Stephens and Krebs, 1986; Hockey, 2013). Behavioral disruptions in either direction are observed in certain human pathologies, including attention deficit hyperactivity disorder, obsessive-compulsive disorder, and depressive disorders (American Psychiatric Association, 2013; Chong et al., 2016; Pessiglione et al., 2018).

Neural activity during the acute decision phase of selecting a high-effort, high-reward course of action has been studied in multiple species, including humans (Croxson et al., 2009; Engstrom et al., 2014; Arulpragasam et al., 2018), laboratory rats (Bardgett et al., 2009; Ostrander et al., 2011; 
Cowen et al., 2012), macaque monkeys (San-Galli et al., 2018), and marmosets (Enomoto et al., 2018). However, there are far fewer studies examining neural activity after the initial decision phase, i.e., what drives an animal to continue to persist in (or quit) an effortful task once the task has been initiated? Neurocognitive frameworks of fatigue suggest that extended effort expenditure recruits functional connectivity between the anterior cingulate cortex (ACC), the anterior insula, and the lateral prefrontal cortex (Muller and Apps, 2019). It is not known though whether modifying activity in any of these regions can alter persistence (or quitting) behaviors in a given task.

The ACC [cingulate cortex area $1(\mathrm{Cg} 1)$ in rodent] is a region with known involvement in motivated behavior. Electrical stimulation of the human ACC at $50 \mathrm{~Hz}$ evokes subjective reports of motivation to accomplish goals and surpass challenges (Parvizi et al., 2013). Similarly, ablation of ACC in humans is sufficient to reduce some of the cognitive symptoms of obsessive-compulsive disorder (Sheth et al., 2013). In laboratory rats, neurons in Cg1 encode effort-outcome values (Hillman and Bilkey, 2010, 2012; Cowen et al., 2012) and manipulations of this region affect an animal's preference for high-effort, high-reward courses of action (Walton et al., 2003; Schweimer and Hauber, 2005, 2006; Rudebeck et al., 2006). Others report, however, that Cg1 activity might mediate some types of effortful action, but not all (Holec et al., 2014). This inconsistency regarding the precise role of the ACC/Cg1 in effort-laden motivated behavior is not surprising given the wide range of phenomena and functions ascribed to the ACC/Cg1, including autonomic regulation, fear and anxiety, nociception, and attention (Medford and Critchley, 2010). ACC/Cg1's involvement in diverse functions suggests it is a major node in high-order cognitive control circuitry, including that required for complex, dynamic decision making (Heilbronner and Hayden, 2016; Kolling et al., 2016; Wang et al., 2018).

Like the ACC, the anterior insula [broadly homologous to the agranular insular (AI) in rodent] is implicated in a wide range of phenomena, including aggression, fear, interoception, frustration, and food- and drug-seeking behaviors (Craig, 2009). Of note, the $\mathrm{AI}$ is involved in mediating behavioral responses to changes in cost-benefit parameters: both lesioning and GABAergic inhibition of AI in rodents promotes the pursuit of higher food outcomes in tasks with reward devaluation schedules (Balleine and Dickinson, 2000; Parkes et al., 2015). These findings have found resonance in similar experiments with cocaine (Moschak et al., 2018) and nicotine (Pushparaj et al., 2013). In human experiments when feelings of frustration are induced by blocking participants' progression in a task, there is coincident activation of a network that includes the anterior insula (Yu et al., 2014). In another human experiment, selfreported feelings of satisfaction after curiosity-inducing tasks are associated with insular activity (Lee and Reeve, 2017). Taken together, these results suggest that the anterior insula plays a central role in controlling internal motivational states which may influence persistence or quitting behaviors.

The ACC and anterior insula have been proposed to functionally interact as a Salience Network (Medford and Critchley, 2010; Menon and Uddin, 2010). This network - which can facilitate network shifts between the Default Mode Network and Central Executive Network - helps an animal appropriately respond to salient cues, whether those cues stem from challenges and changes in environment, expectations, preferences, and/or internal signals (Medford and Critchley, 2010; Scholl et al., 2015). All of these cue types dynamically change during an effort-laden task, suggesting that Salience Network node activity may be critical in driving - or dissuading - persistence in the task at hand.

Here we used a laboratory rat model to investigate whether electrical stimulations of ACC/Cg1 or AI change an animal's persistence in an effortful weightlifting task (WLT). We tested a low $(10 \mathrm{~Hz})$ and a high $(130 \mathrm{~Hz})$ frequency as behavioral effects of stimulation are often influenced by frequency (Mohan et al., 2020). For example in kindled rats, seizure activity can be precipitated with $10 \mathrm{~Hz}$ hippocampal stimulation but suppressed with $130 \mathrm{~Hz}$ (e.g., Wyckhuys et al., 2010). We predicted that $10 \mathrm{~Hz}$ Cg1 stimulation would increase persistence in the WLT given that Cg1 activity in rodent is linked to high-effort, high-reward choice behavior, and that mid-frequency $(50 \mathrm{~Hz})$ pre-operative ACC stimulation in humans has been associated with a "will to persevere" (Parvizi et al., 2013). We predicted that $130 \mathrm{~Hz}$ AI stimulation would reduce task engagement and persistence, given that a previous study linked $130 \mathrm{~Hz}$ insular stimulation to reduced nicotine self-administration in a progressive ratio operant task (Pushparaj et al., 2013).

\section{MATERIALS AND METHODS}

\section{Animals}

Ten male Sprague-Dawley rats $(n=10)$ were sourced from the University of Otago's Hercus Taieri Resource Unit (Dunedin, New Zealand) and housed in $38 \times 30 \times 35 \mathrm{~cm}$ clear plexiglass, individually ventilated cages (Tecniplast, Italy). At the beginning of experiments, rats were approximately 7 months of age with an average body weight of $439 \mathrm{~g}( \pm 5.8 \mathrm{~g})$. At the end of experiments, rats were approximately 10 months of age with an average body weight of $436 \mathrm{~g}$ ( $\pm 5.1 \mathrm{~g})$. Animals were paired in cages but kept separate by a clear, perforated barrier so auditory and olfactory interaction could happen, but no direct physical contact could occur. All animals were kept on a $12 \mathrm{~h}$ reverse dark-light cycle, with all experimental procedures being conducted during the animal's dark phase. The rats were kept on a restricted diet of standard rat chow (Teklad diet, Envigo, United States) to limit their body weight and promote interest in food reward; all rats were maintained at $\geq 85 \%$ of their free-feeding body weight. Water was available ad libtum. All procedures were approved by the Animal Ethics Committee at the University of Otago, protocol 91/17.

\section{Weightlifting Task}

Before surgery, animals were trained in the WLT, a novel effort expenditure task that was recently developed and validated by our lab. For a detailed description of the WLT apparatus, materials, and full training procedures, see Porter and Hillman (2019); videos of the task in action are included in the supplementary material of that publication. In brief, the WLT consists of a $120 \mathrm{~cm} \times 90 \mathrm{~cm} \times 60 \mathrm{~cm}$ wooden open arena, painted black. 

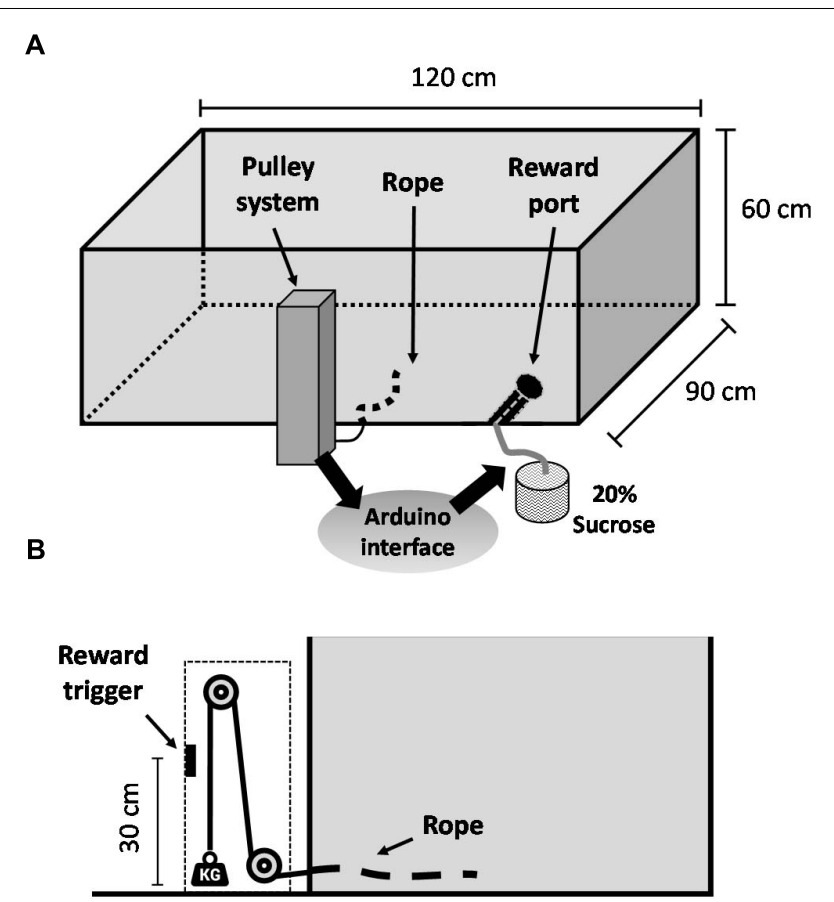

FIGURE 1 | Weightlifting task schematic. (A) General overview of the apparatus. (B) Profile view of the weighted pulley system that allows for effort manipulations within the task. Video footage of the apparatus and of the task in action is included in the supplementary material of Porter and Hillman (2019).

Inside the arena, two conduit pipes extend from the wall: one conduit contains a rope, which is connected outside the arena to a vertical pulley system; the other conduit contains a silicone tube, which is connected outside the arena to a peristaltic pump containing 20\% sucrose liquid (Figure 1A). The animal must pull the rope $30 \mathrm{~cm}$ to trigger automated dispensing of $0.2 \mathrm{ml}$ sucrose reward. The pulley system enables different weights to be added to the rope, ranging 45-225 g in $45 \mathrm{~g}$ increments, thus increasing the difficulty of rope pulling within the arena (Figure 1B). Animals were initially trained on a rope containing no weight ("0 g") and once proficient were challenged with $45 \mathrm{~g}$. Training was considered complete once an animal was able to perform 10 successful pulls of $0 \mathrm{~g}$, immediately followed by 10 successful pulls of $45 \mathrm{~g}$, all within $5 \mathrm{~min}$. The WLT is automated via an Arduino microcontroller, which is configured to send TTL signals to a nearby acquisition system (Digital Lynx SX; Neuralynx Inc.) for timestamping of all task events.

\section{Surgery}

Once trained in the WLT, animals were prepared for surgical implantation of electrodes. Rats were placed in an induction chamber and given 5\% isoflurane in oxygen mixture (EZ-7000, EZ Anesthesia, United States). Once voluntary movement ceased, the animals were removed from the chamber and placed in a stereotaxic frame (Stoelting, United States) equipped with nontraumatic ear bars and a nose cone for anesthetic maintenance at
$2-4 \%$. The animals received subcutaneous doses of amphoprim (trimethoprim and sulphadimethyl pyrimidine, $30 \mathrm{mg} / \mathrm{kg}$ ), atropine $(0.065 \mathrm{mg} / \mathrm{kg})$, and buprenorphine $(0.05 \mathrm{mg} / \mathrm{kg})$. The scalp was infused with subcutaneous bupivacaine $(4 \mathrm{mg} / \mathrm{kg})$. A single incision was made over the scalp and the skull exposed, bregma and lambda were located and the two landmarks were leveled via nose bar adjustment. Craniotomies were drilled for electrode implantations and for the placement of structural screws. Coordinates for the implants, taken from bregma, were: $\mathrm{AP}+3.7, \mathrm{ML}+0.4, \mathrm{DV}-1.0$ for $\mathrm{Cg} 1$; and $\mathrm{AP}+2.7, \mathrm{ML}$ +2.0 , DV -5.8 for the AI, with the stereotaxic arm angled $20^{\circ}$ toward the right from midline. Coordinates were based on the rat atlas of Paxinos and Watson (2007); all implants were right hemisphere. Half the rats received stimulating electrodes in the Cg1, and the other half received stimulating electrodes in the AI. Each animal was also implanted with recording electrodes for local field potential (LFP) capture; animals with stimulating electrodes in Cg1 had ipsilateral recording electrodes in AI, and vice versa. LFP data are not presented in this report. The electrodes were connected to gold pins inside a McIntyre plug which was cemented to skull screws.

Once surgery was finished, animals were administered a dose of carprofen ( $5 \mathrm{mg} / \mathrm{kg}$, s.c.), ear bars were removed, and isoflurane was reduced to $0 \%$. After $10 \mathrm{~min}$ of breathing oxygen mixture through the nose cone, animals were transferred to a clean, sterile cage and were monitored for $6 \mathrm{~h}$. Animals were then returned to their home cage and closely monitored for 7 days; during this time there was free access to food and water. Animals were returned their pre-surgical food restriction regimen on post-surgical day eight.

\section{Electrodes and Stimulation}

Both stimulating and recording electrodes were made of twisted and PFA-insulated stainless steel wires (diameter 0.005" bare, $0.008^{\prime \prime}$ coated, SDR Scientific, Australia). The stimulating wires were twisted together, with each tip separated by $0.5 \mathrm{~mm}$; this spacing was selected to limit current spread to a minimum (Bagshaw and Evans, 1976; Tehovnik, 1996). Recording electrodes were arrays constituted of three wires twisted together. The free ends of each electrode were soldered to gold pins and inserted into a McIntyre connector (Molino and McIntyre, 1972).

Electrical stimulation was generated by an isolated, constant current stimulator (Model 4100, A-M Systems, United States). Stimulation trains consisted of biphasic square pulses (100 ms pulse width, $75 \mu \mathrm{A}$ amplitude); these parameters are based on previous ACC and AI stimulation experiments (Pushparaj et al., 2013; Lim et al., 2015) and preliminary trials in our laboratory. Three frequencies were used separately in different trials: sham stimulation $(0 \mathrm{~Hz})$, low frequency stimulation (LFS, $10 \mathrm{~Hz}$ ), and high frequency stimulation (HFS, $130 \mathrm{~Hz}$ ), which were delivered uninterrupted throughout the testing session. Stimulation sessions were performed with at least $24 \mathrm{~h}$ interval between each session. While in the testing apparatuses, animal movement was tracked via an overhead camera (CV-S3200, JAI, United States) and headstage mounted LEDs. 


\section{Experimental Design}

After surgery and recovery, animals were returned to the WLT apparatus for testing, initially to confirm that they recalled the task and there were no post-surgical impairments in performance. The initial cohort of animals ( $n=4$; two with Cg1 stimulation electrodes and two with AI stimulation electrodes) was run through a counterbalanced A-B-C block design, with blocks of sham, LFS, or HFS. Each block contained 3 days of testing, one session per day. A second cohort $(n=6$; three with $\mathrm{Cg} 1$ stimulation electrodes and three with $\mathrm{AI}$ stimulation electrodes) was run through a counterbalanced A-B-C-C-B-A block design, with blocks of sham, LFS, or HFS. Each block contained 3 days of testing, one session per day.

For each session, the animal was placed in the WLT arena (with no rope initially present), stimulation was initiated, and the animal was given 2 min of arena exploration. After this baseline period, the $0 \mathrm{~g}$ rope was fed into the arena and the animals began the WLT. After 10 successful trials (i.e., pulling the rope $30 \mathrm{~cm}$ to trigger reward dispensing) on the $0 \mathrm{~g}$ rope, a $45 \mathrm{~g}$ weight was attached to the rope. The task continued in this progressive manner - where $45 \mathrm{~g}$ was added every 10 successful trials - until the animal quit the task or reached the max weight of $225 \mathrm{~g}$. A quit was defined as a $60 \mathrm{~s}$ period of being off-task, i.e., no rope pull attempts and no sucrose consumption within $60 \mathrm{~s}$. The weight on which the animal quit the task was deemed the "break weight." When a quit was determined, the experimenter retracted the rope from the arena and the animal was given a final $2 \mathrm{~min}$ of arena exploration. At the end of this 2 min period, two doses of sucrose reward were manually dispensed by the experimenter in order to ascertain if animals quit the task due to satiation. The animal was then returned to its home cage. The floor of the arena was cleaned with a disinfectant liquid (Tego 2001, Hugh Crane, United Kingdom; 1\% solution) in between sessions, and illumination of the experimental room was kept to a minimum.

To determine if stimulation of either brain region affected general locomotor behavior, animals also completed a series of open field recording sessions. These sessions were completed in the weeks following completion of the WLT. The circular open field apparatus was $70 \mathrm{~cm}$ in diameter, $55 \mathrm{~cm}$ high and made of black flexible plastic. The apparatus was placed in the center of the WLT arena, allowing the same recording equipment and the same room parameters (inter-session cleaning, illumination) to be used. Each session was $5 \mathrm{~min}$ in duration, one session per day. Animals were given one initial day of habituation (no stimulation), and then stimulation sessions were carried out using the same block format as was used in the WLT, with counterbalanced blocks of sham, LFS, and HFS.

\section{Perfusion and Histology}

After all experiments were completed, rats were euthanized with isoflurane, and transcardially perfused with saline $(0.9 \%)$ followed by paraformaldehyde solution (4\%) and formalinsucrose solution (30\%). Brains were removed and stored in formalin-sucrose for at least $48 \mathrm{~h}$ to allow fixation and to prepare for frozen sectioning. Sectioning was conducted in a microtomecryostat (Leica CM1860 UV, Germany) at $80 \mu \mathrm{m}$ thickness, and slices were mounted on clear glass slides. Sections were then stained with thionin and digitally captured, and the locations of the recording and stimulating tips were established according to the rat brain atlas of Paxinos and Watson (2007). All animals had stimulating tips confirmed to be within the borders of the target region (Figure 2).

\section{Data Analysis}

Initial data analyses were carried out using Matlab R2018a and custom Matlab scripts. Video tracking and TTL signals exported from the acquisition system were used to calculate behavioral metrics such as trial duration, attempts-to-success ratio, and time-on-task; all metrics have been detailed previously (Porter and Hillman, 2019; Porter et al., 2020). Collated data were then exported to GraphPad Prism 8.4.3 for statistical analyses and graphing.

To determine if behavioral metrics from the overall session differed between stimulation conditions, normality was first assessed by Shapiro-Wilk. Parametric (one-way ANOVA) or non-parametric (Kruskal-Wallis) tests were then used accordingly to compare conditions, with post hoc Dunn's comparisons made for each stimulation condition versus sham. To determine if intra-session metrics (trial duration and attempts-to-success) differed between stimulation conditions, two-way ANOVA tests were used with factors of Stimulation Condition and Pulling Weight, and post hoc Dunnett's. Asterisks are used throughout to denote significant differences as follows: ${ }^{*} p<0.05,{ }^{* *} p<0.01,{ }^{* * *} p<0.001$. Since the WLT is designed to allow an animal to quit at any point, and most animals quit before reaching the highest weights of 180 and $225 \mathrm{~g}$, there was a scarcity of trial data at these higher weights. For this reason, we constrained our trial-based comparative analyses to the first four weights - $0,45,90$, and $135 \mathrm{~g}$ - where all ten animals routinely contributed data.

\section{RESULTS}

\section{Effects of Stimulation on General Motor Behavior}

No overt motoric effects were observed during delivery of LFS or HFS to either brain region (Figure 3). When tested in a circular open field, stimulation of $\mathrm{Cg} 1$ or $\mathrm{AI}$ did not affect distance traveled as compared to sham $[C g 1: F(2,16)=0.51, p=0.61$; AI: $H(2)=0.92, p=0.21$ ]. Speed was no different between sham, LFS and HFS conditions [Cg1: $F(2,14)=0.53, p=0.60$; AI: $F(2,8)=0.97, p=0.42$ ]. Likewise the different stimulation conditions did not differ in thigmotaxis $[C g 1: F(2,16)=0.26$, $p=0.77 ; A I: H(2)=1.6, p=0.47]$ or freezing behavior $[C g 1$ : $F(2,16)=0.12, p=0.89 ; A I: H(2)=1.05, p=0.63]$.

\section{WLT Performance}

The WLT has recently been detailed and validated by our lab using male Sprague-Dawley rats (Porter and Hillman, 2019). Here we used a specific version of the WLT - the progressive WLT - to challenge the animals in terms of effortful persistence. 
A

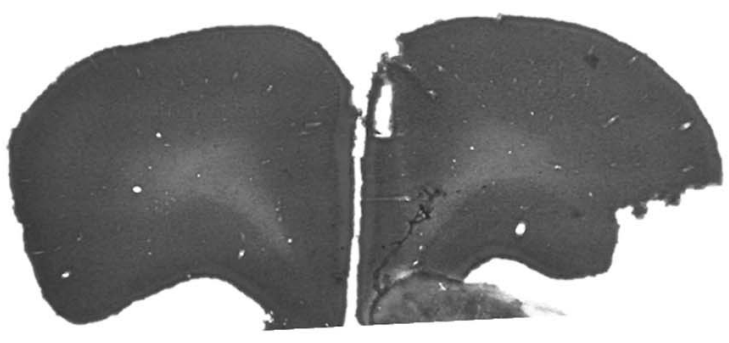

B

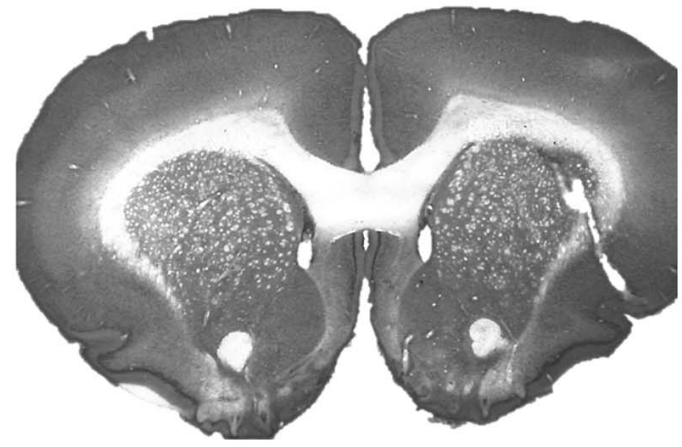

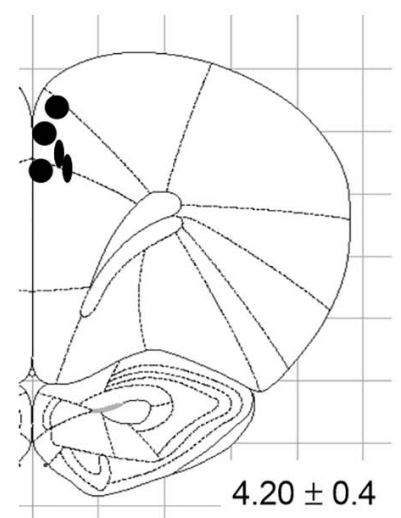

$4.20 \pm 0.4$

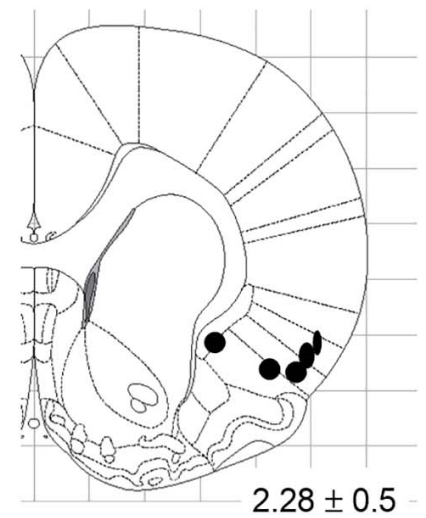

FIGURE 2 I Stimulating electrode placements. (A) Representative coronal slice and schematic illustrating terminal tip locations for Cg1-targeted electrodes. (B) Representative coronal slice and schematic illustrating terminal tip locations for Al-targeted electrodes. Schematics adapted from the Rat Brain Atlas of Paxinos and Watson (2007); distance from bregma indicated in $\mathrm{mm} \pm$ anterior-posterior span.

Rats initially pull a $0 \mathrm{~g}$ rope $30 \mathrm{~cm}$ to trigger a sucrose reward, thereby completing one trial. After every 10 successful trials the rope is weighted with an additional $45 \mathrm{~g}$. This is repeated every 10 successful trials until the animal quits, or reaches a maximum pulling weight of $225 \mathrm{~g}$ (see sections "Weightlifting Task" and "Experimental Design").

Under sham stimulation conditions ( $n=43$ sessions), animals in this study performed the WLT as expected (Figure 4) and behavioral metrics aligned with metrics observed in previous cohorts (Porter and Hillman, 2019; Porter et al., 2020). As the pulling weight progressively increased, the time to complete a successful pull and earn reward (trial duration) significantly increased $[H(3)=50.2, p<0.001]$, as did the attempts-to-success ratio $[H(3)=26.6, p<0.001]$. The most common break weight was $135 \mathrm{~g}$, occurring in $51 \%$ of sham sessions.

\section{Effects of Cg1 Stimulation on WLT Performance}

We predicted that low-frequency Cg1 stimulation would increase persistence in the progressive WLT, that is, stimulation would cause animals to work longer and harder in each task session. Session duration did differ between conditions $[H(2)=9.7$, $p=0.008$; Figure 5A, left], but stimulation did not result in longer WLT sessions. Rather, post hoc comparisons revealed that Cg1 LFS sessions were shorter in duration as compared to sham $(p=0.04)$. Within each session, percentage of time-on-task also differed between conditions $[H(2)=19.4, p<0.001$; Figure 5B, left], but stimulation did not result in more dedicated timeon-task as predicted. Rather, Cg1 HFS produced a decrease in time-on-task $(p=0.03)$.

Despite this decrease in time-on-task under HFS, HFS sessions were associated with higher break weights (Figure 5C, left). When break weight distributions were fitted with Gaussian curves, bidirectional differences in mean break weight were observed in Cg1 stimulation conditions $[F(2,9)=39.9, p<0.001]$. Under Cg1 sham conditions, the most common break weight was $135 \mathrm{~g}$ (occurring in 59\% of Cg1 sham sessions) with a fitted mean $\pm \mathrm{SD}$ of $121 \pm 27 \mathrm{~g}$. Under HFS a rightward shift was observed as compared to sham: the most common HFS break weights were 135 and $180 \mathrm{~g}$ (each occurring in $36 \%$ of HFS sessions) with a fitted mean of $153 \pm 45 \mathrm{~g}$. Under LFS, there was a leftward shift as compared to sham: the most common LFS break weight was $90 \mathrm{~g}$ (occurring in $50 \%$ of LFS sessions) with a fitted mean of $99 \pm 32$. Under Cg1 LFS the animals quit the task sooner - as indicated by 
A

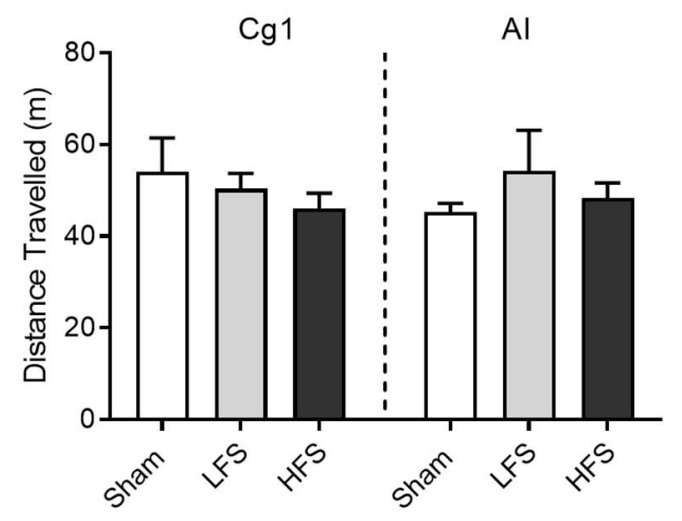

C

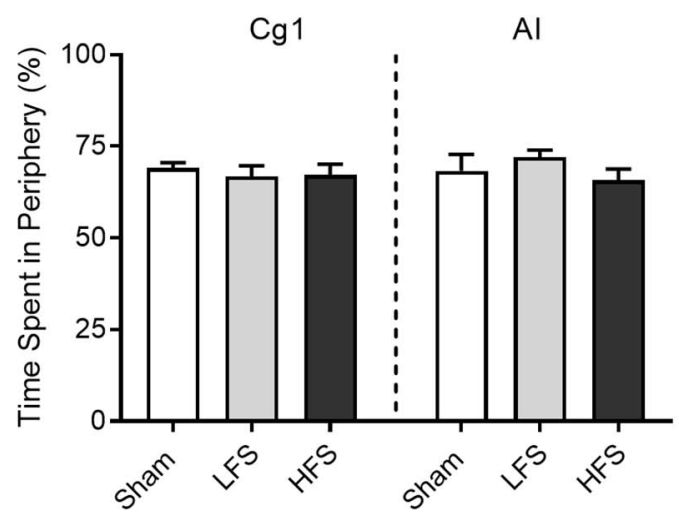

B

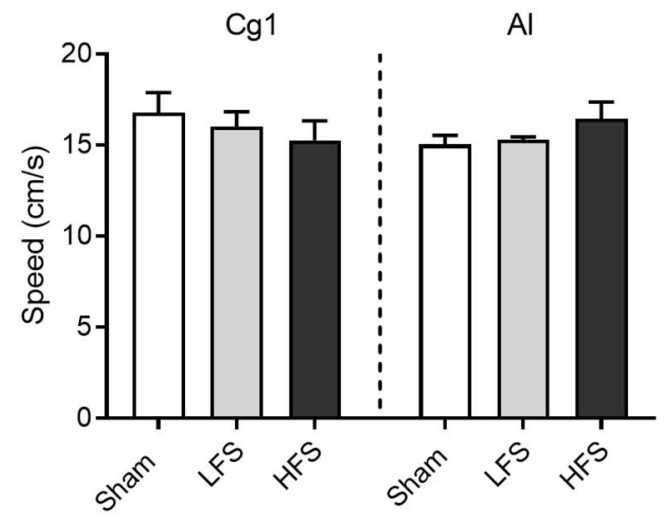

D

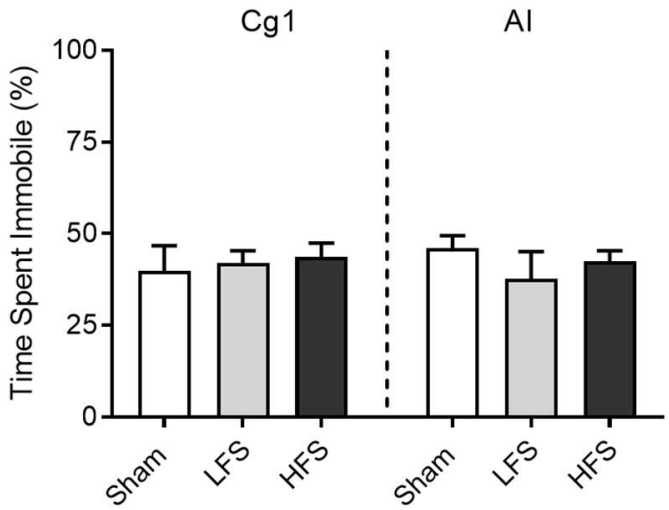

FIGURE 3 | Effects of stimulation on open field behavior. (A) Distance traveled. (B) Average speed across the 5 min session. (C) Time spent in the periphery of the open field, demarcated as within $20 \mathrm{~cm}$ of the apparatus' wall. (D) Time spent immobile, defined as moving speed $<10 \mathrm{~cm} / \mathrm{s}$. Data are shown as mean \pm SEM.

A

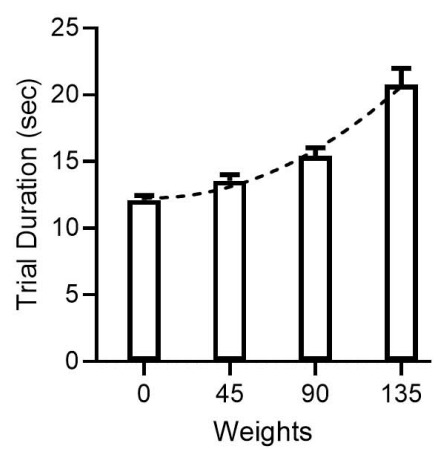

B

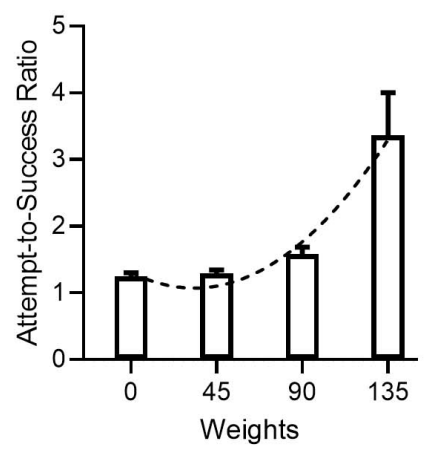

C

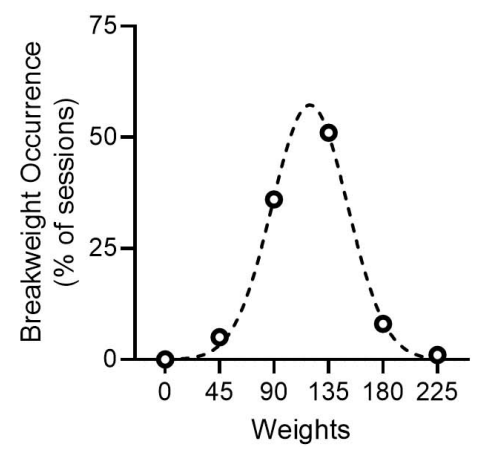

FIGURE 4 | Progressive WLT performance under sham stimulation. (A) Trial duration, with one trial being defined as the time from rope pull initiation to reward consumption. Dotted line denotes second order polynomial. (B) Attempts-to-success ratio. A ratio of one indicates that a single rope pull attempt was successful and resulted in one reward being dispensed; values $>1$ indicate that multiple pull attempts were needed to successfully trigger reward. Dotted line denotes second order polynomial. (C) Break weight distribution across sham sessions. Open circles denote observed frequencies, dotted line denotes Gaussian fit. Data for panel $(\mathbf{A}, \mathbf{B})$ are shown as mean \pm SEM. 
A

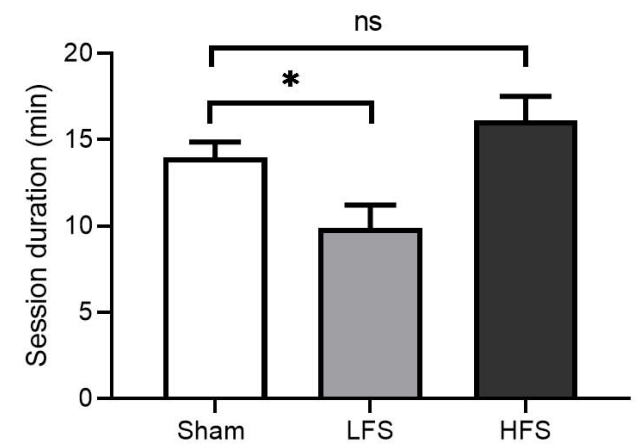

B

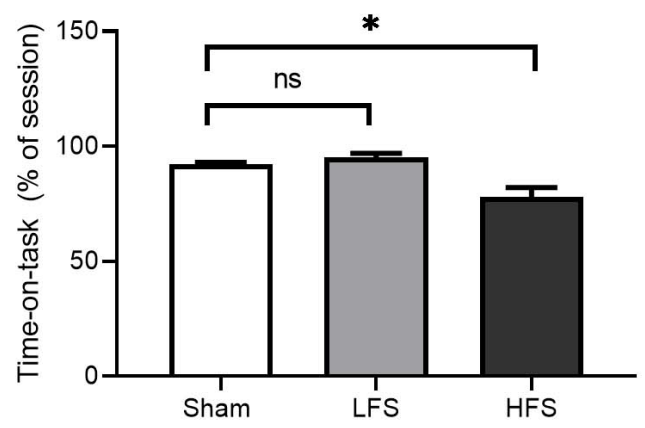

C

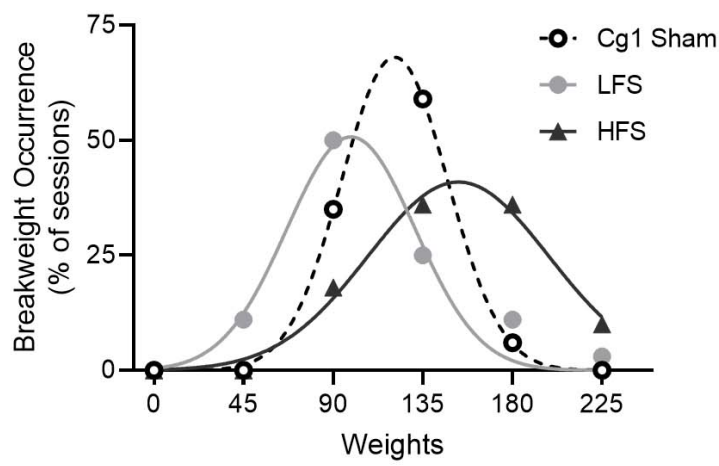

D

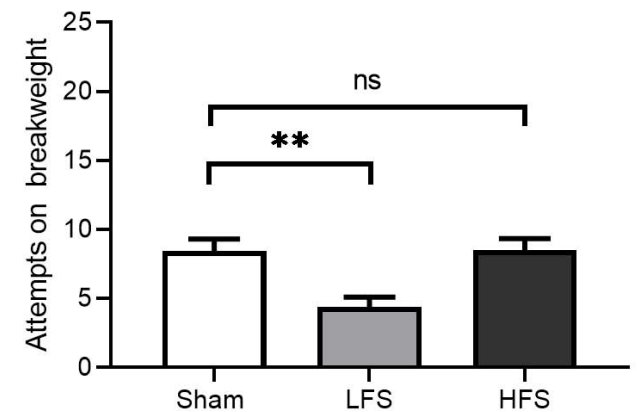

Al Stimulation
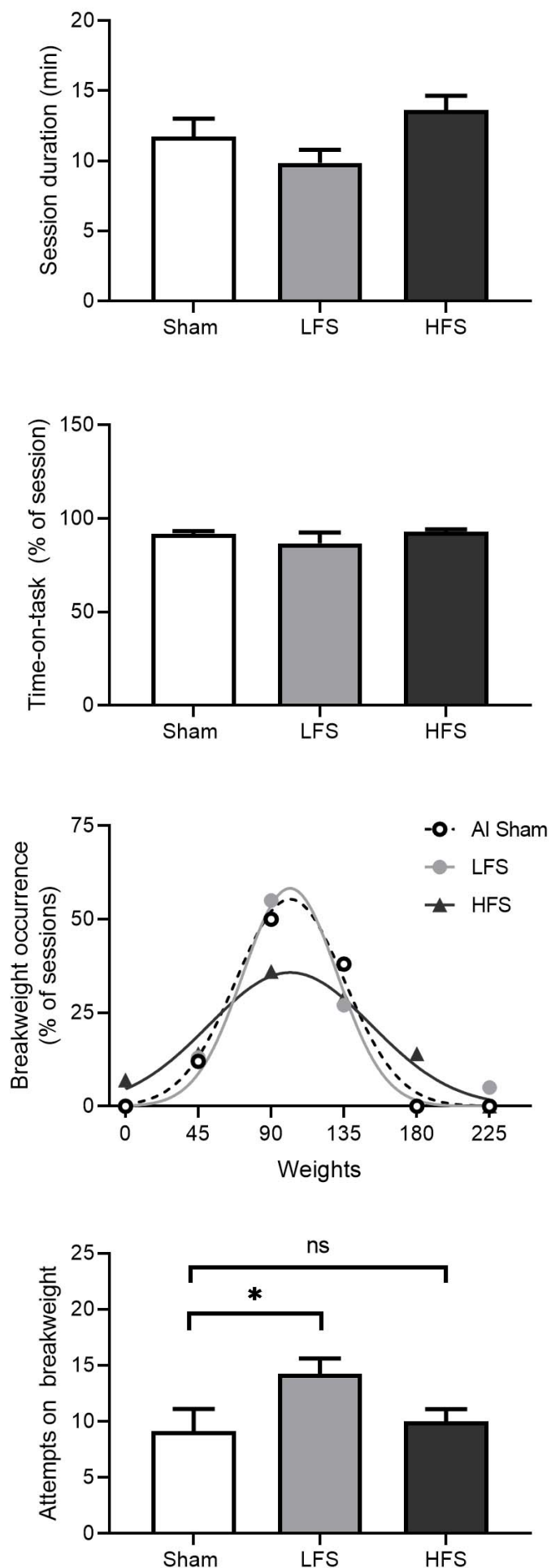

FIGURE 5 | Effects of stimulation on WLT session performance. Cg1 stimulation data are presented on the left, Al stimulation data are presented on the right. (A) Session duration. Animals could quit the task at any time point. (B) Time-on-task, calculated as the percent of session duration engaged in rope pulling and reward consumption. (C) Break weight distribution comparisons between stimulation conditions. Symbols denote observed frequencies, lines denote Gaussian fits. (D) Attempts made on the break weight prior to quitting. For all bar graphs, data are shown as mean \pm SEM. Asterisks denote significant post hoc comparisons as compared to sham; ${ }^{\star} p<0.05,{ }^{\star \star} p<0.01$. 
A

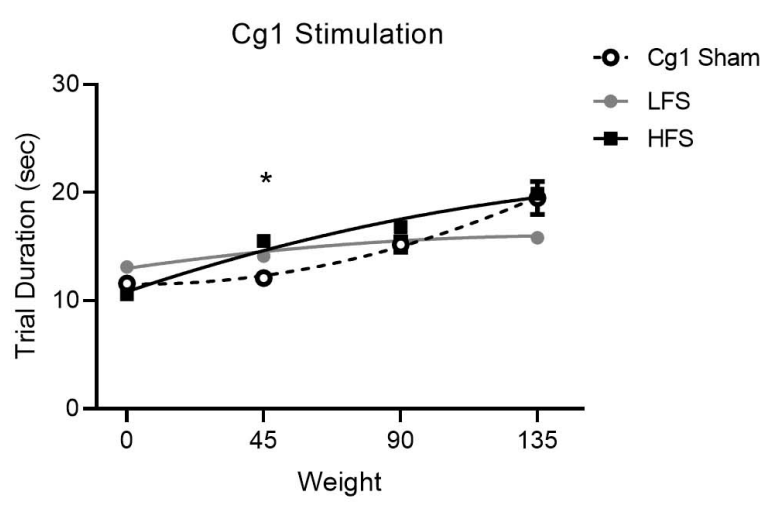

B

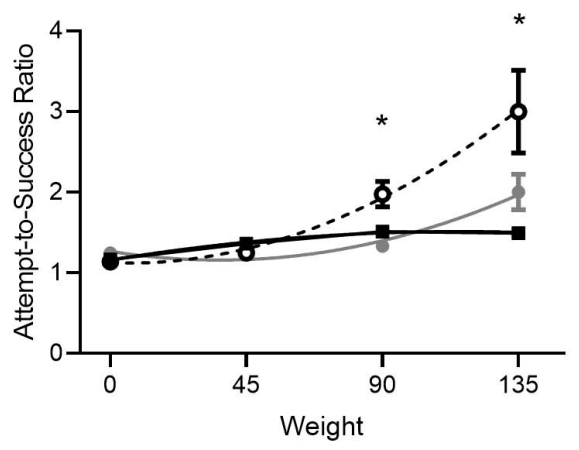

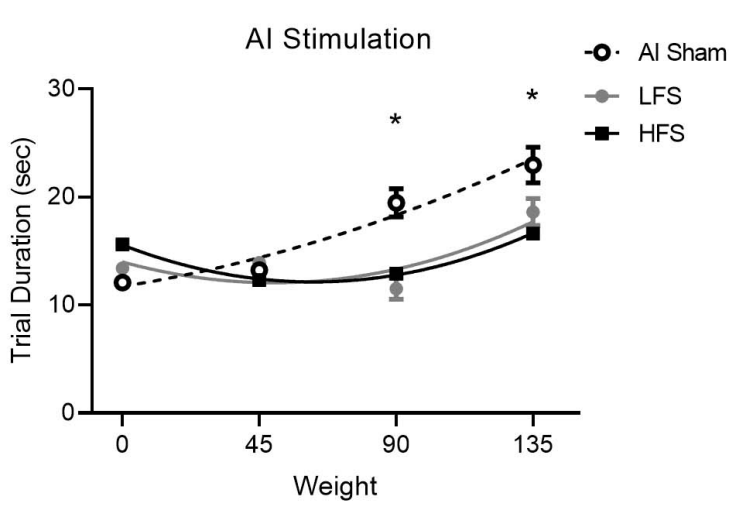

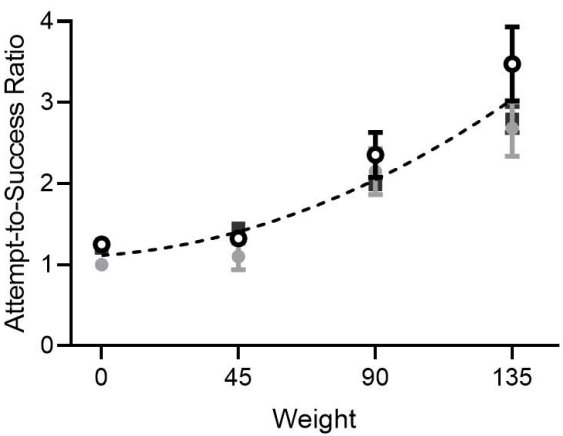

FIGURE 6 | Effect of stimulation on trial performance. Cg1 stimulation data are presented on the left, Al stimulation data are presented on the right. (A) Trial duration for stimulations versus sham. (B) Attempt-to-success ratios for stimulations versus sham. Data symbols denote mean \pm SEM; lines illustrate second order polynomial best fit. For attempts-to-success ratios during insula stimulation there is only one line of best fit illustrated, as all three conditions could be fit with a shared global curve. Asterisks denote significant post hoc comparisons where both stimulation conditions (LFS and HFS) differed from sham.

shorter session durations and lower break weights - and the animals also made fewer attempts on the break weight before electing to quit (Figure 5D, left). When the break weight trials were examined in isolation to determine how many attempts were made before quitting, attempts differed between conditions $[H(2)=12.2, p=0.002]$, with LFS significantly lower than $\operatorname{sham}(p=0.006)$.

To determine if Cg1 stimulation had more subtle effects on performance within the task itself, trial duration and attemptsto-success ratio were examined across the three stimulation conditions (Figure 6, left). Trial duration exhibited a significant Condition $\times$ Weight interaction $[F(6,396)=10.3, p<0.001]$. The data were best represented by distinct quadratic fits $[F(6,399)=10.3, p<0.001]$. When attempts-to-success ratios were examined, a significant Condition $\times$ Weight interaction was also observed $[F(6,396)=5.9, p<0.001]$. Again the different stimulation conditions were best represented by distinct quadratic fits $[F(6,399)=8.4, p<0.001]$.

\section{Effects of Al Stimulation on WLT Performance}

We predicted that AI stimulation would reduce effort investment in the WLT, that is, animals would spend more time off-task and/or quit the task sooner. When overall session durations were compared, no effect of AI stimulation was detected $[H(2)=5.1, p=0.08$; Figure 5A, right $]$. Within each session, percentage of time-on-task also did not differ between conditions $[H(2)=0.39, p=0.82$; Figure 5B, right $]$. When break weight distributions were fitted with Gaussian curves, mean break weights were no different between conditions $[F(2,9)=3.2$, $p=0.08$; Figure 5C, right]. When break weight trials were examined in isolation to determine how many attempts were made before quitting, stimulation did have a small, marginally significant effect $[F(2,42)=3.1, p=0.055$; Figure 5D, right $]$. Lowfrequency stimulation of the AI was associated with an increase in the number of attempts on the break weight as compared to $\operatorname{sham}(p=0.046)$.

To determine if AI stimulation had more subtle effects on performance within the task itself, trial duration and attemptsto-success ratio were examined across conditions (Figure 6, right). Trial duration exhibited a significant Condition $\times$ Weight interaction $[F(6,477)=8.24 .1, p<0.001]$. The data were best represented by distinct quadratic fits $[F(6,480)=8.6$, $p<0.001]$. When attempts-to-success ratios were examined, there was no Condition $\times$ Weight interaction $[F(6,477)=1.1$, $p=0.37]$. The same curve fit could be applied to all conditions $[F(6,480)=1.9, p=0.08]$. 


\section{DISCUSSION}

Given that conjoint activation of the cingulate cortex and insular cortex is observed during effortful decision-making (Engstrom et al., 2014), volitional responding (Medford and Critchley, 2010), and task switching (Menon and Uddin, 2010), we hypothesized that stimulation of these areas in rat would alter performance in an effortful weightlifting task. Despite being used clinically, electrical stimulation in vivo is still not well understood in terms of mechanism. Stimulation can cause proximal as well as distal effects and has been linked to neuronal excitation as well as inhibition; stimulation frequency appears to be one important determinant in these differing effects (Bari et al., 2013; Mohan et al., 2020). For this reason we tested two stimulation frequencies - 10 and $130 \mathrm{~Hz}$ - frequencies which have been used successfully in prior rat studies (Pushparaj et al., 2013; Rea et al., 2014; Lim et al., 2015; Lindenbach et al., 2019). We initially predicted bidirectional effects based on stimulation site: that Cg1 stimulation would increase effort expenditure and persistence in the task, and that AI stimulation would decrease effort expenditure and prompt earlier quitting.

Contrary to our prediction, low-frequency Cg1 stimulation resulted in shorter task sessions: animals quit the task sooner (lower break weight) and made less attempts on the break weight before quitting. One interpretation of this is that LFS reduced motivation to perform the task, notably as it got more difficult, however performance metrics within the session indicated that there was motivated rope-pulling throughout. At 0 and $45 \mathrm{~g}$ LFS was associated with slower trial-by-trial performance (longer trial duration) but the attempts-to-success ratio centered around 1; the latter continued into higher weights indicating that when rats decided to initiate a pull they were generally successful. One interpretation of this is that Cg1 LFS facilitates a "slow and steady" approach, where the time to complete a single trial is slower but the efficiency of the pull is maintained (i.e., an attempt-to-success ratio $\sim 1$ ), even at higher pulling weights. This differs from sham stimulation conditions, where there is an exponential increase in attempts-to-success ratio as the pulling weight increases. Under sham stimulation in this study, and as observed in non-stimulated animal cohorts in previous studies (Porter and Hillman, 2020; Porter et al., 2020), heavier weights result in more failed attempts - i.e., rope pulls fail to reach the $30 \mathrm{~cm}$ mark required to trigger reward. Cg1 LFS appears to exert a subtle change in WLT behavior: the animal experiences fewer fails, but also terminates the task earlier.

High-frequency Cg1 stimulation in our animals was associated with more time spent off-task in each session, however this did not equate to poorer overall performance in the task. Rather, HFS was again associated with a "slow and steady" pulling efficiency but also with a higher break weight. Taken together these data suggest that under Cg1 HFS animals work consistently at the task, even into higher weights, but take frequent small breaks which culminate in more time spent off-task. To our knowledge this is one of the first studies to examine rat Cg1 modulation during an effortful task. Hart et al. (2020) also recently published a study examining Cg1 modulation during an effortful task; they demonstrated that chemogenetic excitation and inhibition of the region reduced lever-pressing in a progressive ratio, choice-based task. Similar to our study, the Hart et al. (2020) findings defy simple interpretation: manipulations assumed to be opposing produced similar behavioral shifts. While puzzling, both studies demonstrate that $\mathrm{Cg} 1$ manipulation during a task can shift effort expenditure in subtle ways.

Electrical stimulation of the rat cingulate and deeper vmPFC has been examined in other previous studies that were framed toward investigating anxiety- and depressive-like behaviors. However, results of those studies have been mixed. For example, Lim et al. (2015) compared the effects of 10 and $100 \mathrm{~Hz}$ $\mathrm{Cg} 1 / \mathrm{vmPFC}$ stimulation in naive Sprague-Dawley males as the animals performed a battery of tests. HFS reduced home cage emergence latency and increased food intake, however null effects were reported in the open field, sucrose intake test and forced swim test. Rea et al. (2014) used $130 \mathrm{~Hz}$ vmPFC stimulation in rats from the Flinders Sensitive Line, a genetic animal model of depression, and found that HFS improved sucrose consumption and forced swim performance. Our findings that LFS and HFS of Cg1 produce subtle but significant changes in WLT performance in naïve animals suggests that the WLT may be worth investigating in future rodent studies investigating anxietyand depressive-like behaviors.

Low-frequency insular stimulation in our study was associated with increased attempts on the break weight and faster performance (shorter trial duration) on higher weights. Faster performance on higher weights was also observed in the HFS condition for AI. In both stimulation conditions, there was no difference in attempts-to-success ratio as compared to sham, and no difference in end break weight or time on-task as compared to sham. Taken together, one interpretation is that AI stimulation (LFS or HFS) increases the speed of the animal's trial-by-trial task performance in high-effort circumstances, but this does not equate to improved efficiency or improved performance overall (i.e., higher break weight). In our open field assessment HFS in the AI initially appeared to induce a slight increase in speed (Figure 3) however this was not a significant difference compared to sham $(p=0.38)$. Thus the change in speed observed in our animals appears task-specific and not a general change in locomotor activity, a finding that has also been observed from stimulation of the lateral habenula in male Wistar rats (Jakobs et al., 2019).

In a previous insular stimulation study in rat, Pushparaj et al. (2013) demonstrated that $130 \mathrm{~Hz}$ HFS decreased nicotine selfadministration in fixed-ratio and progressive-ratio operant tasks. These behavioral results mirrored the group's earlier findings using baclofen inactivation of in the insula (Forget et al., 2010) leading the authors to suggest that HFS is producing a regional inactivation effect. In our study, however, we found no significant effect of AI HFS on progressive-ratio performance overall (i.e., break point) for sucrose reward. Likewise, a prior study using quinolinic acid lesioning of the insula also reported no effect on progressive-ratio responding for normal food pellets (Daniel et al., 2017). Thus it is difficult to interpret just how insula manipulation alters effort expenditure - whether it affects motivation, effort exertion, or more generally the decisionmaking framework. 
Lesioning of the rat anterior insula by various methodologies suggests it may be at the more general level of decisionmaking framework. Optimal choice selection in a slot machine task is reduced following GABAergic inhibition of the insula (Cocker et al., 2016); decision-making in a rodent version of the Iowa Gambling Task is altered following quinolinic lesioning of the insula (Daniel et al., 2017); and strategy shifting in response to sensory specific satiety is reduced following chemogenetic manipulation of the insula (Parkes et al., 2018). Importantly, Daniel et al. (2017) highlighted the impact of the individual rat's baseline behavioral preference pre-insular lesion, and this may be worth considering in regional stimulation studies. Daniel et al. (2017) demonstrated that insular inactivation in baseline "good decision makers" caused a shift toward less-optimal exploitation behavior, whereas insular inactivation in baseline "poor decision makers" caused a shift toward more optimal exploitation. Individual variability in gambling decisions likely explains the discrepant results that other groups reported in insular manipulation gambling studies which analyzed group means (Mizoguchi et al., 2015; Pushparaj et al., 2015). Because of the small sample size in the current study $(n=10)$, we were reliant on group means and could not perform robust group splits into baseline "loweffort" and "high-effort" rats, however this is an area ripe for future investigation.

Finally, for future studies of Cg1/AI stimulation, we offer two methodological considerations. In this study we utilized unilateral stimulation; it would be interesting to repeat the WLT using bilateral stimulation to determine if the subtle effects we observed here become more prominent with bilateral stimulation. Indeed many of the studies discussed above utilized bilateral stimulation (Pushparaj et al., 2013; Rea et al., 2014; Lim et al., 2015; Lindenbach et al., 2019). In this study we also utilized continuous stimulation for the duration of the task

\section{REFERENCES}

American Psychiatric Association (2013). DSM-V: Diagnostic and statistical manual of mental disorders, 5th Edn. Washington, D.C.: American Psychiatric Association.

Arulpragasam, A. R., Cooper, J. A., Nuutinen, M. R., and Treadway, M. T. (2018). Corticoinsular circuits encode subjective value expectation and violation for effortful goal-directed behavior. Proc. Natl. Acad. Sci. U S A. 115, E5233-E5242. doi: $10.1073 /$ pnas. 1800444115

Bagshaw, E. V., and Evans, M. H. (1976). Measurement of Current Spread from Microelectrodes when Stimulating within Nervous-System. Exp. Brain Res. 25, 391-400.

Balleine, B. W., and Dickinson, A. (2000). The effect of lesions of the insular cortex on instrumental conditioning: evidence for a role in incentive memory. J. Neurosci. 20, 8954-8964.

Bardgett, M. E., Depenbrock, M., Downs, N., Points, M., and Green, L. (2009). Dopamine modulates effort-based decision making in rats. Behav. Neurosci. 123, 242-251. doi: 10.1037/a0014625

Bari, B. A., Ollerenshaw, D. R., Millard, D. C., Wang, W., and Stanley, G. B. (2013). Behavioral and electrophysiological effects of cortical microstimulation parameters. PLoS One 8:e82170. doi: 10.1371/journal.pone.0082170

Chong, T. T., Bonnelle, V., and Husain, M. (2016). Quantifying motivation with effort-based decision-making paradigms in health and disease. Prog. Brain Res. 229, 71-100. doi: 10.1016/bs.pbr.2016.05.002 session, similar to the studies discussed above. One innovative stimulation approach has recently been detailed by Lindenbach et al. (2019). Their study examined $20 \mathrm{~Hz}$ stimulation of the ventral subiculum during a progressive-ratio task, but rather than continuous stimulation the researchers applied stimulation only after the first fail ( \pm stimulation at the start of the session). This idea of using stimulation to "boost" performance after a failed attempt lends itself well to the WLT and combined with bilateral stimulation could provide interesting results.

\section{DATA AVAILABILITY STATEMENT}

The raw data supporting the conclusions of this article will be made available by the authors, without undue reservation.

\section{ETHICS STATEMENT}

The animal study was reviewed and approved by the University of Otago Animal Ethics Committee, AUP 91/17.

\section{AUTHOR CONTRIBUTIONS}

KLH and BSP conceived the study. CS ran the experiments. BSP wrote analysis routines. All authors participated in data analysis and manuscript preparation.

\section{FUNDING}

This study was supported by Marsden Fund grant U001617 (KLH) from the Royal Society of New Zealand Te Apārangi.

Cocker, P. J., Lin, M. Y., Barrus, M. M., Le Foll, B., and Winstanley, C. A. (2016). The agranular and granular insula differentially contribute to gambling-like behavior on a rat slot machine task: effects of inactivation and local infusion of a dopamine D4 agonist on reward expectancy. Psychopharmacology 233, 3135-3147. doi: 10.1007/s00213-016-4355-1

Cowen, S. L., Davis, G. A., and Nitz, D. A. (2012). Anterior cingulate neurons in the rat map anticipated effort and reward to their associated action sequences. J. Neurophysiol. 107, 2393-2407. doi: 10.1152/jn.01012.2011

Craig, A. D. (2009). How Do You Feel - Now? The Anterior Insula and Human Awareness. Nat. Rev. Neurosci. 10, 59-70. doi: 10.1038/nrn2555

Croxson, P. L., Walton, M. E., O'Reilly, J. X., Behrens, T. E., and Rushworth, M. F. (2009). Effort-based cost-benefit valuation and the human brain. J. Neurosci. 29, 4531-4541. doi: 10.1523/JNEUROSCI.4515-08.2009

Daniel, M. L., Cocker, P. J., Lacoste, J., Mar, A. C., Houeto, J. L., Belin-Rauscent, A., et al. (2017). The anterior insula bidirectionally modulates cost-benefit decision-making on a rodent gambling task. Eur. J. Neurosci. 46, 2620-2628. doi: 10.1111/ejn.13689

Engstrom, M., Karlsson, T., Landtblom, A. M., and Craig, A. D. (2014). Evidence of Conjoint Activation of the Anterior Insular and Cingulate Cortices during Effortful Tasks. Front. Hum. Neurosci. 8:1071. doi: 10.3389/fnhum.2014.01071

Enomoto, T., Konoike, N., Takemoto, A., Nakamura, K., and Ikeda, K. (2018). Blockade of dopamine $\mathrm{D}(1)$ receptors, but not $\mathrm{D}(2)$ receptors, decreases motivation in a novel effort-discounting paradigm in common marmosets. Behav. Neurosci. 132, 526-535. doi: 10.1037/bne0000273 
Forget, B., Pushparaj, A., and Le Foll, B. (2010). Granular insular cortex inactivation as a novel therapeutic strategy for nicotine addiction. Biol. Psychiatry 68, 265-271. doi: 10.1016/j.biopsych.2010.01.029

Hart, E. E., Blair, G. J., O’Dell, T. J., Blair, H. T., and Izquierdo, A. (2020). Chemogenetic Modulation and Single-Photon Calcium Imaging in Anterior Cingulate Cortex Reveal a Mechanism for Effort-Based Decisions. J. Neurosci. 40, 5628-5643. doi: 10.1523/JNEUROSCI.2548-19.2020

Heilbronner, S. R., and Hayden, B. Y. (2016). Dorsal Anterior Cingulate Cortex: A Bottom-Up View. Annu. Rev. Neurosci. 39, 149-170. doi: 10.1146/annurevneuro-070815-013952

Hillman, K. L., and Bilkey, D. K. (2010). Neurons in the rat anterior cingulate cortex dynamically encode cost-benefit in a spatial decision-making task. J. Neurosci. 30, 7705-7713. doi: 10.1523/JNEUROSCI.1273-10.2010

Hillman, K. L., and Bilkey, D. K. (2012). Neural encoding of competitive effort in the anterior cingulate cortex. Nat. Neurosci. 15, 1290-1297. doi: 10.1038/nn. 3187

Hockey, R. (2013). The Psychology of Fatigue. Cambridge: Cambridge University Press.

Holec, V., Pirot, H. L., and Euston, D. R. (2014). Not all effort is equal: the role of the anterior cingulate cortex in different forms of effort-reward decisions. Front. Behav. Neurosci. 8:12. doi: 10.3389/fnbeh.2014.00012

Hull, C. L. (1943). Principles of behavior: an introduction to behavior theory. Oxford: Appleton-Century.

Jakobs, M., Pitzer, C., Sartorius, A., Unterberg, A., and Kiening, K. (2019). Acute 5 $\mathrm{Hz}$ deep brain stimulation of the lateral habenula is associated with depressivelike behavior in male wild-type Wistar rats. Brain Res. 1721:146283. doi: 10 . 1016/j.brainres.2019.06.002

Kolling, N., Behrens, T., Wittmann, M. K., and Rushworth, M. (2016). Multiple signals in anterior cingulate cortex. Curr. Opin. Neurobiol. 37, 36-43. doi: 10.1016/j.conb.2015.12.007

Lee, W., and Reeve, J. (2017). Identifying the neural substrates of intrinsic motivation during task performance. Cogn. Affect. Behav. Neurosci. 17, 939953. doi: 10.3758/s13415-017-0524-x

Lim, L. W., Prickaerts, J., Huguet, G., Kadar, E., Hartung, H., Sharp, T., et al. (2015). Electrical stimulation alleviates depressive-like behaviors of rats: investigation of brain targets and potential mechanisms. Transl. Psychiat. 5:24. doi: 10.1038/ tp. 2015.24

Lindenbach, D., Seamans, J. K., and Phillips, A. G. (2019). Activation of the ventral subiculum reinvigorates behavior after failure to achieve a goal: Implications for dopaminergic modulation of motivational processes. Behav. Brain Res. 356, 266-270. doi: 10.1016/j.bbr.2018.09.002

Medford, N., and Critchley, H. D. (2010). Conjoint activity of anterior insular and anterior cingulate cortex: awareness and response. Brain Struct. Funct. 214, 535-549. doi: 10.1007/s00429-010-0265-x

Menon, V., and Uddin, L. Q. (2010). Saliency, switching, attention and control: a network model of insula function. Brain Struct. Funct. 214, 655-667. doi: 10.1007/s00429-010-0262-0

Mizoguchi, H., Katahira, K., Inutsuka, A., Fukumoto, K., Nakamura, A., Wang, T., et al. (2015). Insular neural system controls decision-making in healthy and methamphetamine-treated rats. Proc. Natl. Acad. Sci. U S A. 112, E3930-E3939. doi: 10.1073/pnas.1418014112

Mohan, U. R., Watrous, A. J., Miller, J. F., Lega, B. C., Sperling, M. R., Worrell, G. A., et al. (2020). The effects of direct brain stimulation in humans depend on frequency, amplitude, and white-matter proximity. Brain Stimul. 13, 1183 1195. doi: 10.1016/j.brs.2020.05.009

Molino, A., and McIntyre, D. C. (1972). Another inexpensive headplug for the electrical recording and or stimulation of rats. Physiol. Behav. 9, 273-275. doi: 10.1016/0031-9384(72)90249-1

Moschak, T. M., Wang, X., and Carelli, R. M. (2018). A Neuronal Ensemble in the Rostral Agranular Insula Tracks Cocaine-Induced Devaluation of Natural Reward and Predicts Cocaine Seeking. J. Neurosci. 38, 8463-8472. doi: 10.1523/ JNEUROSCI.1195-18.2018

Muller, T., and Apps, M. A. J. (2019). Motivational fatigue: A neurocognitive framework for the impact of effortful exertion on subsequent motivation. Neuropsychologia 123, 141-151. doi: 10.1016/j.neuropsychologia.2018. 04.030

Ostrander, S., Cazares, V. A., Kim, C., Cheung, S., Gonzalez, I., and Izquierdo, A. (2011). Orbitofrontal cortex and basolateral amygdala lesions result in suboptimal and dissociable reward choices on cue-guided effort in rats. Behav. Neurosci. 125, 350-359. doi: 10.1037/a0023574

Parkes, S. L., Bradfield, L. A., and Balleine, B. W. (2015). Interaction of insular cortex and ventral striatum mediates the effect of incentive memory on choice between goal-directed actions. J. Neurosci. 35, 6464-6471. doi: 10.1523/ JNEUROSCI.4153-14.2015

Parkes, S. L., Ravassard, P. M., Cerpa, J. C., Wolff, M., Ferreira, G., and Coutureau, E. (2018). Insular and Ventrolateral Orbitofrontal Cortices Differentially Contribute to Goal-Directed Behavior in Rodents. Cereb. Cortex 28, 2313-2325. doi: $10.1093 /$ cercor/bhx132

Parvizi, J., Rangarajan, V., Shirer, W. R., Desai, N., and Greicius, M. D. (2013). The Will to Persevere Induced by Electrical Stimulation of the Human Cingulate Gyrus. Neuron 80, 1359-1367. doi: 10.1016/j.neuron.2013.10.057

Paxinos, G., and Watson, C. (2007). The Rat Brain in Stereotaxic Coordinates. Amsterdam: Academic Press.

Pessiglione, M., Vinckier, F., Bouret, S., Daunizeau, J., and Le Bouc, R. (2018). Why not try harder? Computational approach to motivation deficits in neuropsychiatric diseases. Brain 141, 629-650. doi: 10.1093/brain/awx278

Porter, B. S., and Hillman, K. L. (2020). Dorsomedial prefrontal neural ensembles reflect changes in task utility. bioRxiv 2020:370536. doi: 10.1101/2020.11.05. 370536

Porter, B. S., Li, K., and Hillman, K. L. (2020). Regional Activity in the Rat Anterior Cingulate Cortex and Insula during Persistence and Quitting in a Physical-Effort Task. eNeuro 7:2020. doi: 10.1523/ENEURO.0243-20.2020

Porter, B., and Hillman, K. L. (2019). A Novel Weight Lifting Task for Investigating Effort and Persistence in Rats. Front. Behav. Neurosci. 13:275. doi: 10.3389/ fnbeh.2019.00275

Pushparaj, A., Hamani, C., Yu, W., Shin, D. S., Kang, B., Nobrega, J. N., et al. (2013). Electrical Stimulation of the Insular Region Attenuates Nicotine-Taking and Nicotine-Seeking Behaviors. Neuropsychopharmacol 38, 690-698. doi: 10.1038/ npp.2012.235

Pushparaj, A., Kim, A. S., Musiol, M., Zangen, A., Daskalakis, Z. J., Zack, M., et al. (2015). Differential Involvement of the Agranular vs Granular Insular Cortex in the Acquisition and Performance of Choice Behavior in a Rodent Gambling Task. Neuropsychopharmacol 40, 2832-2842. doi: 10.1038/npp.2015.133

Rea, E., Rummel, J., Schmidt, T. T., Hadar, R., Heinz, A., Mathe, A. A., et al. (2014). Anti-anhedonic effect of deep brain stimulation of the prefrontal cortex and the dopaminergic reward system in a genetic rat model of depression: an intracranial self-stimulation paradigm study. Brain Stimul. 7, 21-28. doi: 10.1016/j.brs.2013.09.002

Rudebeck, P. H., Walton, M. E., Smyth, A. N., Bannerman, D. M., and Rushworth, M. F. (2006). Separate neural pathways process different decision costs. Nat. Neurosci. 9, 1161-1168. doi: 10.1038/nn1756

San-Galli, A., Varazzani, C., Abitbol, R., Pessiglione, M., and Bouret, S. (2018). Primate Ventromedial Prefrontal Cortex Neurons Continuously Encode the Willingness to Engage in Reward-Directed Behavior. Cereb. Cortex 28, 73-89. doi: 10.1093/cercor/bhw351

Scholl, J., Kolling, N., Nelissen, N., Wittmann, M. K., Harmer, C. J., and Rushworth, M. F. (2015). The Good, the Bad, and the Irrelevant: Neural Mechanisms of Learning Real and Hypothetical Rewards and Effort. J. Neurosci. 35, 11233 11251. doi: 10.1523/JNEUROSCI.0396-15.2015

Schweimer, J., and Hauber, W. (2005). Involvement of the rat anterior cingulate cortex in control of instrumental responses guided by reward expectancy. Learn. Mem. 12, 334-342. doi: 10.1101/lm.90605

Schweimer, J., and Hauber, W. (2006). Dopamine D1 receptors in the anterior cingulate cortex regulate effort-based decision making. Learn. Mem. 13, 777 782. doi: 10.1101/lm.409306

Sheth, S. A., Neal, J., Tangherlini, F., Mian, M. K., Gentil, A., Cosgrove, G. R., et al. (2013). Limbic system surgery for treatment-refractory obsessive-compulsive disorder: a prospective long-term follow-up of 64 patients. J. Neurosurg. 118, 491-497. doi: 10.3171/2012.11.JNS12389

Stephens, D. W., and Krebs, J. R. (1986). Foraging Theory. Princeton: Princeton University Press.

Tehovnik, E. J. (1996). Electrical stimulation of neural tissue to evoke behavioral responses. J. Neurosci. Meth. 65, 1-17. doi: 10.1016/0165-0270(95)00131-X

Walton, M. E., Bannerman, D. M., Alterescu, K., and Rushworth, M. F. S. (2003). Functional specialization within medial frontal cortex of the anterior cingulate for evaluating effort-related decisions. J. Neurosci. 23, 6475-6479. 
Wang, J. X., Kurth-Nelson, Z., Kumaran, D., Tirumala, D., Soyer, H., Leibo, J. Z., et al. (2018). Prefrontal cortex as a meta-reinforcement learning system. Nat. Neurosci. 21, 860-868. doi: 10.1038/s41593-018-0147-8

Wyckhuys, T., Raedt, R., Vonck, K., Wadman, W., and Boon, P. (2010). Comparison of hippocampal Deep Brain Stimulation with high $(130 \mathrm{~Hz})$ and low frequency $(5 \mathrm{~Hz})$ on afterdischarges in kindled rats. Epilepsy Res. 88, 239246. doi: 10.1016/j.eplepsyres.2009.11.014

Yu, R., Mobbs, D., Seymour, B., Rowe, J. B., and Calder, A. J. (2014). The neural signature of escalating frustration in humans. Cortex 54, 165-178. doi: 10.1016/ j.cortex.2014.02.013
Conflict of Interest: The authors declare that the research was conducted in the absence of any commercial or financial relationships that could be construed as a potential conflict of interest.

Copyright $\odot 2021$ Silva, Porter and Hillman. This is an open-access article distributed under the terms of the Creative Commons Attribution License (CC BY). The use, distribution or reproduction in other forums is permitted, provided the original author(s) and the copyright owner(s) are credited and that the original publication in this journal is cited, in accordance with accepted academic practice. No use, distribution or reproduction is permitted which does not comply with these terms. 